\title{
THE ASYMPTOTIC DENSITY OF SEQUENCES
}

\section{IVAN NIVEN}

1. Introduction. Our purpose is to outline the recent work on the asymptotic or limit density of sets of positive integers, and to give further details of some recently announced results $[45 ; 46] .{ }^{1} \S \S 2-7$ are concerned with the first objective, and $\S \S 8-12$ with the second. The related concept of Schnirelmann density is touched upon, but we mention only the high spots of work on this topic, including basic sequences and essential components.

In the case of many sequences, much more than the mere density is known. The prime number theorem, for example, implies that the set of primes has density zero, but it tells much more. However, asymptotic estimates are not available for many sequences, and for these it is of interest to know the density. In selecting examples of sequences of density zero in $\S 11$, as applications of the results of $\S 8$, we have tried to choose sets for which analytic estimates are not at present known. We are indebted to $H$. S. Zuckerman for valuable suggestions concerning the formulations in $\S 8$.

2. Definitions. The sequence $A$ of positive integers $a_{1}<a_{2}<\cdots$ has lower density $\delta_{1}(A)$ and upper density $\delta_{2}(A)$ defined by

$$
\delta_{1}(A)=\liminf _{n \rightarrow \infty} \frac{A(n)}{n}, \quad \delta_{2}(A)=\limsup _{n \rightarrow \infty} \frac{A(n)}{n},
$$

where $A(n)$ denotes the number of integers of $A$ which are not greater than $n$. The value $\delta_{1}(A)$ has been referred to variously as the asymptotic density, limit density, or density of $A$. In this paper, however, we shall say that $A$ has a density $\delta(A)$ only if $\delta_{1}(A)=\delta_{2}(A)$, in which case we can write

$$
\delta(A)=\lim _{n \rightarrow \infty} \frac{A(n)}{n} .
$$

This is sometimes called the natural density of $A$. The assertion that almost all positive integers have a certain property $\mathrm{P}$ means that $\delta(A)=1$, where $A$ is the set of integers having the property $\mathrm{P}$. It can be established that for infinite sequences $\delta_{1}(A)=\lim \inf n / a_{n}$,

An address delivered before the Pullman meeting of the Society on June 16, 1951 by invitation of the Committee to Select Hour Speakers for Far Western Sectional Meetings; received by the editors June 20, 1951.

${ }^{1}$ Numbers in brackets refer to the references at the end of the paper. 
$\delta_{2}(A)=\lim \sup n / a_{n}$, so that $\delta(A)=\lim n / a_{n}$ if this limit exists. The Schnirelmann density $d(A)$ of the sequence $A$ is defined by

$$
d(A)=\text { g.l.b. } \frac{A(n)}{n}
$$

It can be easily proved $\left[53\right.$, p. 202] that $d(A)>0$ if and only if $\delta_{1}(A)$ $>0$ and $1 \in A$.

We shall not concern ourselves here with other definitions of density, such as the variation of Schnirelmann density employed by Besicovitch [6], or the maximum and minimum densities of Pólya [51].

3. The sum of sets. The sum $A+B$ of two sequences is defined as the set of all integers of the form $a, b$, or $a+b$ where $a \in A$ and $b \in B$. The conjecture of Khintchine [36] that $d(A+B) \geqq d(A)+d(B)$ or $d(A+B)=1$ was proved by Mann [41]; in fact Mann proved somewhat more than this. Other formulations of Mann's proof and generalizations of his result have been given by Artin and Scherk [1], Dyson [18], and van der Corput [13]. Cheo [10] has given an example to show that the extension of the Mann theorem to higher dimensions (for example, the two-dimensional Gaussian integers) fails. Details concerning the history of this so-called $\alpha+\beta$ problem, and references to earlier work, can be found in various expository papers $[30 ; 48 ; 53]$.

It is readily proved $[53$, p. 206] that if $d(A)+d(B) \geqq 1$ then $d(A+B)=1$; the analogous theorem [53, p. 207] for lower density is that if $\delta_{1}(A)+\delta_{1}(B)=1$, then there exists an integer $m$ such that $n \in A+B$ if $n>m$. Suppose that we are given non-negative real numbers $\alpha, \beta$, and $\gamma$ satisfying $0 \leqq \alpha+\beta \leqq \gamma \leqq 1$; the question arises whether there exist sets $A$ and $B$ such that $d(A)=\alpha, d(B)=\beta$, and $d(A+B)=\gamma$. This has been answered affirmatively in recent work $[9 ; 39 ; 50]$. As yet unsolved is the corresponding problem for lower density, which can be formulated as follows: for sets $A$ of lower density $\alpha, B$ of lower density $\beta$, what is the range of possible values of $\delta_{1}(A+B)$ ? Certain inequalities have been obtained, as we now indicate.

The analogue of the $\alpha+\beta$ theorem of Mann for lower density is false. We formulate the known results in terms of the set $C$ of all elements of the form $a+b$ with $a \in A, b \in B$. Admit 0 as a possible element of any set $A$, but do not count it in $A(n)$. Thus $C=A+B$ if $0 \in A$ and $0 \in B$. Erdös [27] proved that $\delta_{1}(C) \geqq \delta_{1}(A)+\delta_{1}(B) / 2$ under the hypotheses $0 \in A, 0,1 \in B, \delta_{1}(B) \leqq \delta_{1}(A)$, and $\delta_{1}(A)+\delta_{1}(B) \leqq 1$, 
generalizing an earlier result [26] for the case $A=B$. Shapiro [61] removed the hypothesis $\delta_{1}(B) \leqq \delta_{1}(A)$ by use of the Mann theorem. Ostmann [49] also removed this asymmetric hypothesis, and, replacing the hypothesis $0,1 \in B$ by the assumption that $B$ contain $k$ consecutive integers, established the generalization $\delta_{1}(C) \geqq \delta_{1}(A)$ $+\left(1-k^{-1}\right) \delta_{1}(B)$. Mann [42] has recently obtained an inequality connecting the lower densities of $B$ and $C$ and a modified Schnirelmann density of $A$.

4. Basic sequences, essential components, and the primes. A basic sequence $A$ is one for which there exists a fixed integer $h$ such that every positive integer is a sum of at most $h$ elements of $A$. A sequence $A$ is called an essential component if, for every sequence $B$ with $1>d(B)=\beta>0, d(A+B) \geqq \beta+\phi(\beta)$ where $\phi(\beta)$ depends only on $\beta$. Erdös [22; or 38, Theorem 96 ] has proved that every basic sequence is an essential component. The converse of this is false, by an example of Linnik [40]. Schnirelmann [58; or 38, Theorem 91] has proved that the sequence $P$ consisting of 1 and all primes is basic, in fact that $d(P+P)>0$.

Let $K$ be the collection of $k$ th powers of the positive integers for a fixed integer $k>1$, so that $d(K)=0$. Romanoff [54; or 38, Theorem 103] proved that $d(K+P)>0$, and Davenport and Heilbronn [17] showed that $\delta(K+P)=1$. Next let $H$ be the set of non-negative powers of a fixed integer $h$. Romanoff [54; or 38, Theorem 106] established that $d(H+P)>0$. Write $r(h)$ for $\delta_{1}(H+P)$; Landau [37] proved that $\lim _{h \rightarrow \infty} r(h) \log h=1$. In the case $h=2$, van der Corput [14] showed that $\delta_{2}(H+P)<1$.

5. Sequences without progressions. Various writers have discussed sequences no one of whose members is divisible by any other; let $G=\left\{g_{i}\right\}$ denote such a set. Behrend [3] obtained an asymptotic bound for the partial sums of $\sum g_{i}^{-1}$. It had been conjectured that any such set would have density zero, but Besicovitch [5] constructed a set $G_{1}$ with $\delta_{2}\left(G_{1}\right)>1 / 4$ and $\delta_{1}\left(G_{1}\right)=0$. Erdös [24] generalized a result of Besicovitch, and proved [21] that any $G$ sequence must have lower density zero, that is, $\delta_{1}(G)=0$. More recently Erdös [28] established a necessary and sufficient condition on the elements of $G$ such that $\delta(H)$ exists, $H$ being the set of all multiples of the integers of $G$.

Let $J$ be a sequence no three of whose terms are in arithmetric progression. It had been conjectured that $J(n)=O\left(n^{\alpha}\right)$ for some $\alpha<1$, but Salem and Spencer [56] disproved this, later adding a sequel [57]. Their inequality on $J(n)$ was improved by Behrend [4], who 
constructed a sequence $J$ for which

$$
J(n)>n^{1-c /(\log n) 1 / 2} \quad \text { as } n \rightarrow \infty .
$$

Erdös and Turán [31] proved that any $J$ sequence must satisfy $J(n)<(3 / 8+\epsilon) n$ for $n>n(\epsilon)$, and Moser [44] improved this to $J(n)<5+4 n / 11$ for all $n$. Whether every $J$ sequence must have density zero, or even lower density zero, is an unsolved problem.

6. Other special sequences. Let $A_{1}$ be the set of abundant numbers, that is, positive integers $n$ satisfying $\sigma(n) \geqq 2 n, \sigma(n)$ being the sum of all divisors of $n$. Behrend [2] showed that $.241<\delta_{1}\left(A_{1}\right)$ $\leqq \delta_{2}\left(A_{1}\right)<.332$. Davenport [15] proved that $\delta\left(A_{1}\right)$ exists, as did also Chowla [11] and Erdös [19]. The integer $n$ is said to be $k$-abundant if $\sigma(n) \geqq k n$. Davenport [15] established that the density of $k$-abundant numbers exists and is a continuous function of the real number $k \geqq 1$.

Denoting the number of divisors of $m$ by $\sigma_{0}(m)$, let $A_{2}$ be the sequence of integers $m$ for which $\sigma_{0}(m+1)>\sigma_{0}(m)$. Erdös [23] proved Chowla's conjecture that $\delta\left(A_{2}\right)=1 / 2$, and that integers of the form $p_{1}^{2}+p_{2}^{2}-p_{3}^{2}-p_{4}^{2}$ have positive lower density [25], the $p_{i}$ being primes.

The set $A_{3}$ of integers not divisible by any of the nonzero integers $a_{1}, a_{2}, \cdots, a_{m}$ has density

$$
\begin{aligned}
\delta\left(A_{8}\right)=1 & -\sum_{i} \frac{1}{a_{i}}+\sum_{i<j} \frac{1}{\left[a_{i}, a_{j}\right]}-\sum_{i<j<k} \frac{1}{\left[a_{i}, a_{j}, a_{k}\right]}+\cdots \\
& +\frac{(-1)^{m}}{\left[a_{1}, a_{2}, \cdots, a_{m}\right]},
\end{aligned}
$$

where the brackets denote least common multiple. Heilbronn [34] and Rohrbach [52] proved that

$$
\delta\left(A_{3}\right) \geqq \prod_{i=1}^{m}\left(1-a_{i}^{-1}\right) .
$$

Consider a fixed integer $m=p_{1}^{\alpha_{1}} p_{2}^{\alpha_{2}} \cdots p_{r}^{\alpha_{r}}$ with every $\alpha_{i}>1$, the $p_{i}$ being different primes. Let $B_{m}$ denote the sequence of integers $n$ such that $n / m$ is a square-free integer that is relatively prime to $m$. Define $B_{1}$ to be the square-free integers. Schoenberg [59], studying the Fourier transforms of distribution functions, proved that

$$
\delta\left(B_{m}\right)=\frac{6}{m \pi^{2}} \prod_{i=1}^{r}\left(1+\frac{1}{p_{i}}\right)^{-1},
$$

and indicated that this gave another method to obtain a result of 
Feller and Tornier [32], that the density of integers which are divisible by an even number of squares of different primes is

$$
\frac{1}{2}+\frac{1}{2} \prod_{i=1}^{\infty}\left(1-2 p_{i}^{-2}\right)
$$

the product being over all primes. The Schoenberg result for $\delta\left(B_{1}\right)$ is the well known value $6 / \pi^{2}$ for the density of the square-free integers. Details and references concerning density theorems obtained by analytic study of distribution problems of additive arithmetic functions can be found in an expository paper by Kac [35].

Fogels [33] proved that the (rational) integers which are uniquely factorable (apart from units and the order of the factors) in the quadratic field $R\left((-5)^{1 / 2}\right)$, have density zero. A natural density can be defined for a set of quadratic integers of $R\left((-5)^{1 / 2}\right)$, using the norm to get a counting device. Then the uniquely factorable integers of this particular quadratic field have zero density.

For nonzero integers $a_{1}, a_{2}, \cdots, a_{m}$, say that $n \in A_{4}$ provided that all of $n^{2}+a_{1}, n^{2}+a_{2}, \cdots, n^{2}+a_{m}$ are square-free. Mirsky [43] proved that either $A_{4}$ is empty or $\delta_{1}\left(A_{4}\right)>0$. S. Selberg [60] showed that integers of the form $a x^{2}+b y^{2}+c^{2^{2}}$ have positive lower density, where $a, b$, and $c>1$ are positive.

Let $p(n)$ denote the greatest prime factor of $n$. Chowla and Todd [12] have discussed the sequence $A_{5}$ of integers $n$ for which $p(n)$ $<2 n^{1 / 2}$, and more generally the sequence $A_{6}$ for which $p(n)>c n^{\alpha}$ with $c>1$ and $1 / 2 \leqq \alpha<1$. They prove that $\delta\left(A_{5}\right)=1-\log 2$, and indicate that the same technique yields $\delta\left(A_{6}\right)=-\log \alpha$.

Davenport and Erdös [16] have related the lower density and the logarithmic density $\lim _{n \rightarrow \infty}\left\{\sum_{a_{i} \leqq n} a_{i}^{-1}\right\} / \log n$ of a sequence whose terms are divisible by at least one integer from a fixed sequence. Erdös [20] gave some results on the existence of natural densities of certain sequences defined in terms of additive and multiplicative functions.

Salem and Spencer [55] proved theorems concerning the density of sequences having gaps defined by certain analytic conditions.

7. Measure. None of the densities $\delta, \delta_{1}$, or $\delta_{2}$ is a measure. Disjoint sequences $A$ and $B$ can be readily constructed so that $\delta_{1}(A \cup B)$ $\neq \delta_{1}(A)+\delta_{1}(B)$ and $\delta_{2}(A \cup B) \neq \delta_{2}(A)+\delta_{2}(B)$; for example let $A$ be any set not having a density, and let $B$ be the complement of $A$. As to the density $\delta$, sequences $A$ and $B$ can be constructed $[7, \mathrm{p} .571]$ so that $\delta(A)$ and $\delta(B)$ exist but $\delta(A \cup B)$ and $\delta(A \cap B)$ do not.

R. C. Buck [7] has formulated a measure and a class of measur- 
able sequences, and later added a sequel [8] with E. F. Buck. Let $\mathcal{D}_{0}$ denote the class of sequences which are unions of a finite number of arithmetic progressions, or which differ from some such union by a finite set. Clearly any set in $\mathcal{D}_{0}$ has a density. For any sequence $S$, define $\mu(S)=\lim \inf \delta(A)$ for all $A \in \mathcal{D}_{0}$ such that $A \supset S$. The class $\mathcal{D}_{\mu}$ of measurable sets is defined as all sets $S$ such that $\mu(S)+\mu\left(S^{\prime}\right)=1$, where $S^{\prime}$ denotes the complement of $S$. The class $\mathcal{D}_{0}$ is a proper subset of $\mathcal{D}_{\mu}$, which in turn is a proper subset of the class of sequences possessing a density. The measure of any set is its natural density: if $S \in \mathcal{D}_{\mu}$, then $\mu(S)=\delta(S)$.

Buck proved that $\mathcal{D}_{0}$ is a proper subset of $\mathcal{D}_{\mu}$ by exhibiting infinite sequences, for example $\left\{n^{2}\right\}$, which have measure zero but which do not belong to $\mathcal{D}_{0}$. From a number theoretic point of view, it would be of interest to know whether some of the nontrivial sets having positive density are members of $\mathcal{D}_{\mu}$.

8. Upper density. For any prime $p$ let $A_{p}$ denote the subset of integers $n$ of $A$ for which $p \mid n$ but $p^{2} \nmid n$.

THEOREM 1. If $\left\{p_{i}\right\}$ is a set of primes such that $\sum p_{i}^{-1}=\infty$, then $\delta_{2}(A) \leqq \sum \delta_{2}\left(A_{p_{i}}\right)$ for any sequence $A$.

Proof. Let $I$ denote the set of all positive integers, and let $B^{r}$ be the complement of the set

$$
I_{p_{1}} \cup I_{p_{2}} \cup \cdots \cup I_{p_{r}}
$$

Thus $B^{r}$ is the set of all integers except those $n$ for which $p_{i} \mid n$ but $p_{i}^{2} \nmid n$ for at least one of the primes $p_{1}, p_{2}, \cdots, p_{r}$. Now the number $B^{r}(x)$ of integers in $B^{r}$ which do not exceed $x$ can be seen by induction on $r$ to be

$$
\sum(-1)^{\alpha_{1}+\alpha_{2}+\cdots+\alpha_{r}}\left[\frac{x}{p_{1}^{\alpha_{1}} p_{2}^{\alpha_{2}} \cdots p_{r}^{\alpha_{r}}}\right],
$$

where the sum extends over the $3^{r}$ terms obtained by taking each $\alpha_{i}$ equal to 0,1 , or 2 . The removal of the brackets changes the value of each term by something less than 1 , so we have

$$
B^{r}(x)<3^{r}+\sum(-1)^{\alpha_{1}+\alpha_{2}+\cdots+\alpha_{r}} \frac{x}{p_{1}^{\alpha_{1}} p_{2}^{\alpha_{2}} \cdots p_{r}^{\alpha_{r}}},
$$

or

$$
\frac{B^{r}(x)}{x}<\frac{3^{r}}{x}+\prod_{i=1}^{r}\left(1-p_{i}^{-1}+p^{-2}\right) .
$$


Now the divergence of $\sum p_{i}^{-1}$ implies the divergence of $\sum\left(p_{i}^{-1}-p_{i}^{-2}\right)$, and thus we can choose $r$ sufficiently large so that

$$
\prod_{i=1}^{r}\left(1-p_{i}^{-1}+p_{i}^{-2}\right)<\frac{\epsilon}{2}
$$

for any specified $\epsilon>0$. Next choose $N$ sufficiently large that $3^{r} / x<\epsilon / 2$ for $x \geqq N$. Hence (4), (5), and (6) imply that

$$
\frac{A(x)}{x} \leqq \frac{B^{r}(x)}{x}+\sum_{i=1}^{r} \frac{A_{p_{i}}(x)}{x}<\epsilon+\sum_{i=1}^{r} \frac{A_{p_{i}}(x)}{x},
$$

from which the theorem follows.

Corollary 1. If for a set of primes $\left\{p_{i}\right\}$ we have $\delta\left(A_{p_{i}}\right)=0$ for every $i$, and if $\sum p_{i}^{-1}=\infty$, then $\delta(A)=0$.

Feller and Tornier [32, p. 216] have proved the related result that if $\sum p_{i}^{-1}=\infty$, then the sequence of integers divisible by at most $k$ of these primes has density zero, for fixed $k$. This is implied by Corollary 1 ; in fact, we have the following slightly stronger formulation.

Corollary 2. For any fixed $k$, if $\left\{p_{i}\right\}$ is a set of primes for which $\sum p_{i}^{-1}=\infty$, and if $A$ is any sequence whose members are divisible by at most $k$ of these primes to the first degree, then $\delta(A)=0$.

PROoF. We use induction on $k$, assuming the truth of the result in the case $k-1$. Denote the set $\left\{p_{i}\right\}$ by $P$. Then the set $A_{p_{i}}$ has density zero because its members are divisible by at most $k-1$ primes to the first degree chosen from the set $P-p_{i}$ (set theoretic subtraction). Thus $\delta(A)=0$ by Corollary 1 .

Corollary 3. Let $\left\{p_{i}\right\}$ be a set of primes for which $\sum p_{i}^{-1}=\infty$. If $\delta_{2}(A) \neq 0$, then $\sum_{i=1}^{\infty} \delta_{2}\left(A_{p_{i}}\right)=\infty$.

Proof. If $\sum_{i=1}^{\infty} \delta_{2}\left(A_{p_{i}}\right) \neq \infty$, then for any given $\epsilon>0$ there exists an $m$ such that $\sum_{i=m}^{\infty} \delta_{2}\left(A_{p_{i}}\right)<\epsilon$. But $\sum_{i=m}^{\infty} p_{i}^{-1}$ diverges, so by Theorem 1 we have $\delta_{2}(A)<\epsilon$. Hence $\delta_{2}(A)=0$, contrary to hypothesis.

9. Lower density. We now show that the results of the last section are false for lower density, by exhibiting a set $A$ for which $\delta_{1}(A)$ $=1 / 2$ but $\delta_{1}\left(A_{p}\right)=0$ for every prime $p$. Let $\rho_{k}$ denote the $k$ th prime, $k=1,2, \cdots$. Now any positive integer $n$ can be located between successive binomial coefficients as follows: $C(m, 2) \leqq n<C(m+1,2)$. Define the positive integer $f=f(n)=n+1-C(m, 2)$, so that $f$ is a singlevalued function of $n$, whereas to each value of $f$ there correspond infinitely many values of $n$. For any positive integer $n$, define $S^{n}$ as 
the set of integers $x$ satisfying the inequality $n ! \leqq x<(n+1)$ !. Define $T^{n}$ as the set $S^{n}$ with any integer deleted if it is divisible by $\rho_{f}$, where $f=f(n)$ as just defined. Finally, define $A$ as the set obtained by taking the union of the $T^{n}$, thus

$$
A=T^{1} \cup T^{2} \cup T^{3} \cup \ldots .
$$

To evaluate $\delta_{1}(A)$, we note that $S^{n}$ is a finite set of $(n+1) !-n$ ! integers, that not more than half of these integers are deleted from $S^{n}$ to obtain $T^{n}$, so that $T^{n}$ contains at least $\{(n+1) !-n !\} / 2$ integers. Also the $S^{n}$ are disjoint and exhaust $I$, so that $\delta_{1}(A) \geqq 1 / 2$. In case $n$ is of the form $C(m, 2)$ we have $f=f(n)=1$, and $T^{n}$ has exactly $\{(n+1) !-n !\} / 2$ integers. Thus $\delta_{1}(A)=1 / 2$.

On the other hand, for any prime $\rho_{f}$ we consider the sets $T^{n}$ for each of the infinite number of values of $n$ for which $f=f(n)$ as defined above. Such sets $T^{n}$ consist of those integers $x$ in the range $n ! \leqq x<(n+1)$ ! for which $\rho_{f} \nmid x$. Hence we have

$$
\begin{gathered}
A_{\rho f}((n+1) !)-A_{\rho f}(n !)=0, \\
\frac{A_{\rho f}((n+1) !)}{(n+1) !}=\frac{A_{\rho f}(n !)}{n !} \cdot \frac{1}{n+1},
\end{gathered}
$$

from which it follows that $\delta_{1}\left(A_{\text {ps }}\right)=0$.

10. The hypothesis of Theorem 1. For any set of primes $\left\{p_{i}\right\}$ for which $\sum p_{i}^{-1}$ converges, we now exhibit a set $A$ such that $\delta\left(A_{p_{i}}\right)=0$ for all $i$, but $\delta_{2}(A)>0$, so that $\delta(A)>0$ if it exists. Thus the hypothesis $\sum p_{i}^{-1}=\infty$ is essential in Theorem 1 and its corollaries. Define $A$ as the sequence of all integers except those divisible by at least one of the set of primes $\left\{p_{i}\right\}$, so that $\delta\left(A_{p_{i}}\right)=0$ for all $i$. We may presume that the set $\left\{p_{i}\right\}$ is infinite, since otherwise it is clear that $\delta_{2}(A)>0$.

Since $\sum p_{i}^{-1}$ converges, so the product $\Pi\left(1-p_{i}^{-1}\right)$ converges, say to a value $k>0$. Choose $r$ sufficiently large so that $\sum_{i=r+1}^{\infty} p_{i}^{-1}<k / 4$ and then choose $m$ sufficiently large so that $2^{r} / m<k / 4$. Then for any $n \geqq m$ we have

$$
A(n) \geqq \sum(-1)^{\alpha_{1}+\alpha_{2}+\cdots+\alpha_{r}}\left[\frac{n}{p_{1}^{\alpha_{1}} p_{2}^{\alpha_{2}} \cdots p_{r}^{\alpha_{r}}}\right]-\sum_{i=r+1}^{\infty}\left[\frac{n}{p_{i}}\right],
$$

where the first sum ranges over the $2^{r}$ terms obtained by taking each $\alpha_{i}$ equal to 0 or 1 . Thus we have, on removal of brackets,

$$
A(n)>-2^{r}+\sum(-1)^{\alpha_{1}+\alpha_{2}+\cdots+\alpha_{r}} \frac{n}{p_{1}^{\alpha_{1} p_{2}^{\alpha_{2}} \cdots p_{r}^{\alpha_{r}}}}-\sum_{i=r+1}^{\infty} \frac{n}{p_{i}},
$$




$$
\begin{aligned}
\frac{A(n)}{n} & >-\frac{2^{r}}{n}+\prod_{i=1}^{r}\left(1-p_{i}^{-1}\right)-\sum_{i=r+1}^{\infty} p_{i}^{-1} \\
& >-\frac{k}{4}+k-\frac{k}{4}=\frac{k}{2},
\end{aligned}
$$

for all $n \geqq m$. Thus $\delta_{2}(A)>k / 2>0$.

11. Sets of density zero.

THEOREM 2. The multinomial coefficients

$$
\frac{n !}{a_{1} ! a_{2} ! \cdots a_{r} !},
$$

where $n=\sum_{j=1}^{r} a_{j}$ and each $a_{j}<n-1$, have density zero.

Proof. The restriction $a_{j}<n-1$ is needed, for otherwise any integer $n$ can be put in form (7) by choosing $r=2, a_{1}=1, a_{2}=n-1$. There is no loss of generality in assuming that

$$
a_{1} \leqq a_{2} \leqq \cdots \leqq a_{r}
$$

We give two lemmas, in which $\rho_{m}$ denotes the $m$ th prime and $C(n, r)$ the binomial coefficients.

LEMmA 1. If $2 \leqq r \leqq n-r$, there exist positive constants $N$ and $c<1$ such that

$$
C(n, r)>c \rho_{r} \rho_{n-r} \text { for all } n, \quad C(n, r)>\rho_{r} \rho_{n-r} \text { for } n>N .
$$

Proof. We prove that $C(n, r)>\rho_{r} \rho_{n-r}$ for $n$ sufficiently large. Let $k$ be a constant such that $\rho_{m}<k m \log m$ for all $m$. In case $3 \leqq r \leqq n-r$ we have for $n$ sufficiently large,

$$
\begin{aligned}
C(n, r) & \geqq n(n-1)(n-2) / 6>n(n-1)(k \log n)^{2} \\
& >k r \log r \cdot k(n-r) \log (n-r)>\rho_{r} \rho_{n-r .}
\end{aligned}
$$

In case $r=2$ we have for $n$ sufficiently large,

$$
C(n, r)=3 n(n-1) / 6>3 n(k \log n)>3 \rho_{n}>\rho_{2} \rho_{n-2},
$$

which completes the proof of the lemma.

LEMMA 2. If $a_{1}=a_{2}=\cdots=a_{s-1}=1$ and $a_{s}>1$, then $(7)$ is greater than

$$
c^{N} \rho_{1}^{-1} \prod_{j=s}^{r} \rho_{a_{j}}^{2}
$$

where the dash on the product indicates that the first and last terms, $\rho_{a}$ 
and $\rho_{a_{r}}$, are not squared.

Proof. In case $a_{1}>1$ so that $s=1$, we write (7) in the form

$$
C\left(n, a_{r}\right) C\left(n-a_{r}, a_{r-1}\right) C\left(n-a_{r}-a_{r-1}, a_{r-2}\right) \cdots C\left(n-\sum_{j=3}^{r} a_{j}, a_{2}\right) .
$$

By Lemma 1 this exceeds

$$
\rho_{a_{r}} \rho_{n-a_{r}} \rho_{a_{r-1}} \rho_{n-a_{r}-a_{r-1}} \cdots \rho_{a_{2}} \rho_{n}-\Sigma_{2}^{r} a_{j} c^{N},
$$

since the constant $c$ enters in at most $N$ cases. This can be seen to be larger than (9), by replacing $\rho_{n-a_{r}}$ by $\rho_{a_{r-1}}, \rho_{n-a_{r}-a_{r-1}}$ by $\rho_{a_{r-2}}$, and so on.

In case $s>1$, we write (7) in the form

$$
n(n-1) \cdots(n-s+2) \frac{(n-s+1) !}{\prod_{j=s}^{r} a_{j} !},
$$

which by the previous case exceeds

$$
n(n-1) \cdots(n-s+2) c{ }^{N} \prod_{j=8}^{r}{ }^{\prime} \rho_{a_{j}}^{2},
$$

and this is larger than (9), and so the lemma is proved.

We now establish a one-to-one correspondence between the integers (7) and the integers

$$
\rho_{1}^{8-1} \prod_{j=8}^{r} \rho_{a_{j}}^{2}
$$

with the conventions of notation as in Lemma 2. Different sets of values $\left(a_{1}, a_{2}, \cdots, a_{r}\right)$ in (7) yield different integers (10), and the integers (10) have density zero by Corollary 2. Thus by Lemma 2 we complete the proof of Theorem 2, since the $c^{N}$ factor in (9) is irrelevant, being independent of (7).

Theorem 3. The set $A$ of integers $\sigma_{s}(n)$ for all $s \geqq 1$ and all $n \geqq 1$ has density zero, $\sigma_{s}(n)$ being the sum of the sth powers of all divisors of $n$.

Proof. We separate $A$ into two possibly overlapping parts $B$ and $C, \sigma_{s}(n)$ being put in $B$ if $n$ has more than $k$ distinct prime factors, otherwise in $C$. We shall specify $k$ in a moment. For any given $\epsilon>0$ we prove that $A(m)<\epsilon m$ for all sufficiently large $m$.

Any member of the set $B$ satisfies the inequality 


$$
\sigma_{s}(n) \geqq n^{s} \prod_{j=1}^{k}\left(1+\rho_{j}^{-8}\right)=n^{8} c_{8},
$$

$\rho_{j}$ being the $j$ th prime. The last equality defines $c_{s}$, a function of $s$ and $k$, and we choose $k$ so that $\epsilon c_{1}>4$. Thus for fixed $s$ the number of $\sigma_{s}(m)$ counted by $B(m)$ is not more than $\left(m / c_{s}\right)^{1 / s}$, and hence

$$
B(m) \leqq m / c_{1}+\left(m / c_{2}\right)^{1 / 2}+\cdots+\left(m / c_{r}\right)^{1 / r},
$$

where $r \leqq \log _{2} m$ because for any larger value of $r, \sigma_{r}(2)>m$. Now $\left(m / c_{s}\right)^{1 / \bullet}<m^{1 / 2}$ for $s=2,3, \cdots, r$, and so we have

$$
B(m)<m / c_{1}+m^{1 / 2} \log _{2} m .
$$

Turning now to the set $C$, we observe that $\sigma_{s}(n)$ is counted by $C(m)$ provided that $n$ has $k$ or fewer prime factors, and that $\sigma_{s}(n) \leqq m$. Since $\sigma_{s}(n) \geqq n^{s}$, this implies that $n^{s} \leqq m$ or $n \leqq m^{1 / s}$. Let $F$ designate the sequence of integers having $k$ or fewer prime factors. Thus we have

$$
C(m) \leqq \sum_{s=1}^{r} F\left(m^{1 / s}\right),
$$

again with $r \leqq \log _{2} m$. Now $F$ is a set of density zero (by Corollary 2 , for example) so that there exists an $n_{1}$ such that $F(m)<\epsilon m / 2$ for $m>n_{1}$. Also $F\left(m^{1 / s}\right) \leqq m^{1 / 2}$ for $s=2,3, \cdots, r$, and consequently

$$
C(m)<\epsilon m / 2+m^{1 / 2} \log _{2} m .
$$

This with (11) gives

$$
A(m) \leqq B(m)+C(m)<m / c_{1}+\epsilon m / 2+2 m^{1 / 2} \log _{2} m,
$$

and $A(m) / m<c_{1}^{-1}+\epsilon / 2+2 m^{-1 / 2} \log _{2} m$. The last factor is less than $\epsilon / 4$ for $m>n_{2}$, say, so that for $m>\max \left(n_{1}, n_{2}\right)$ we have, recalling that $\epsilon c_{1}>4$,

$$
\frac{A(m)}{m}<\frac{\epsilon}{4}+\frac{\epsilon}{2}+\frac{\epsilon}{4}=\epsilon .
$$

But $\epsilon$ is an arbitrary positive number, so $A$ has density zero.

THEOREM 4. For fixed $k$, the set $A$ of integers $n$ such that $(n, \phi(n)) \leqq k$ has density zero, where $\phi(n)$ is the Euler function.

Proof. It clearly suffices to prove that the set $B$ of integers $n$ such that $(n, \phi(n))=k$ has density zero, since the union of a finite number of sets of density zero also has density zero. Let $\left\{p_{i}\right\}$ be the set of 
primes not dividing $k$, so that $\sum p_{i}^{-1}=\infty$. Now any integer in $B_{p_{i}}$ has the form $m p_{i}$, with $\left(m, p_{i}\right)=1$, and furthermore $\left(\phi(m), p_{i}\right)=1$ since otherwise $p_{i} \mid k$. Hence $m$ has no divisor of the form $y p_{i}+1$. But the primes $\left\{q_{j}\right\}$ of this form have the property $\sum q_{j}^{-1}=\infty$, so that by Corollary 1 the set of permissible values of $m$ has density zero, so that $\delta\left(B_{p_{i}}\right)=0$. Applying Corollary 1 again, we get $\delta(B)=0$.

For $k=1$, the set $A$ of Theorem 4 consists of those integers $n$ with $(n, \phi(n))=1$, and has been studied by Erdös [29], who proved that

$$
A(n)=(1+o(1)) n e^{-\gamma}(\log \log \log n)^{-1} .
$$

Theorem 5. For fixed $m$, the set $A$ of integers a for which $x^{2} \equiv m$ $(\bmod a)$ is solvable has density zero if and only if $m$ is not a perfect square.

Proof. If $m$ is a perfect square, say $m=k^{2}$, then $x \equiv k(\bmod a)$ is a solution of the quadratic congruence. If $m$ is not a perfect square, then $m$ must be a quadratic residue of any prime which divides $a$. But the Gaussian reciprocity law and the Dirichlet theorem imply the existence of infinitely many primes $\left\{p_{i}\right\}$ in an arithmetic progression, each prime having $m$ as a quadratic nonresidue. In fact $\sum p_{i}^{-1}$ diverges, so that $\delta(A)=0$ by Corollary 1 .

12. A binary operation on sequences. If $A$ and $B$ denote sequences of integers $\left\{a_{i}\right\}$ and $\left\{b_{i}\right\}$ respectively, we define the "product" $A B$ to be the set $\left\{b_{a_{i}}\right\}$. Under this operation, sets of integers form an associative, noncommutative groupoid.

THEOREM 6. $\delta_{1}(A B) \geqq \delta_{1}(A) \delta_{1}(B)$.

Proof. We have

$\delta_{1}(A B)=\lim \inf n / b_{a_{n}} \geqq \lim \inf n / a_{n} \cdot \lim \inf a_{n} / b_{a_{n}} \geqq \delta_{1}(A) \delta_{1}(B)$.

In case the densities of $A$ and $B$ exist, then the above proof holds with equality signs throughout, so that $\delta(A B)=\delta(A) \cdot \delta(B)$. If $\delta(A)$ $=0$, then $\delta(A B)=0$ for any set $B$; likewise if $\delta(B)=0$, then $\delta(A B)=0$ for any set $A$.

TheOREM 7. If $\delta(A B)$ exists, and if either $\delta(A)$ or $\delta(B)$ exists and is positive, then the other one exists.

Proof. If $\delta(B)$ exists and is positive, then $\lim b_{a_{n}} / a_{n}$ exists as $n \rightarrow \infty$, and by hypothesis $\lim n / b_{a_{n}}$ exists, and so $\lim n / a_{n}$ exists. On the other hand, if $\delta(A)$ exists and is positive, we denote $A B$ by $C$ and note that $C\left(b_{n}\right)=A(n)$. Thus $n / b_{n}=\{n / A(n)\} \cdot\left\{C\left(b_{n}\right) / b_{n}\right\}$, and so $\lim n / b_{n}$ exists. 
THEOREM 8. For Schnirelmann density, $d(A B) \geqq d(A) d(B)$.

Proof. Denote $d(A)$ by $\alpha, d(B)$ by $\beta$, and $A B$ by $C$. We shall establish that $C(x) \geqq \alpha \beta x$ for all integers $x$ such that $x+1 \in C$, and this implies the theorem. Thus we write

$$
\begin{aligned}
\alpha \beta\left(b_{a_{n}}-1\right) & \leqq \alpha B\left(b_{a_{n}}-1\right)=\alpha\left(a_{n}-1\right) \\
& \leqq A\left(a_{n}-1\right)=n-1=C\left(b_{a_{n}}-1\right),
\end{aligned}
$$

and the proof is complete.

Finally, we cite an example to show that inequalities cannot be removed in Theorems 6 and 8. Let $A$ consist of 1 and all integers $x$ satisfying $2^{n} \leqq x \leqq 2^{n}+2^{n-1}-1$ for $n \geqq 2$. Let $B$ consist of 1 and all $x$ satisfying $2^{n}+2^{n-1} \leqq x \leqq 2^{n+1}-1$ for $n \geqq 2$. Then $A B$ consists of 1 and all $x$ such that $3 \cdot 2^{n} \leqq x \leqq 3 \cdot 2^{n}+2^{n-1}-1$ for $n \geqq 2$, and $B A$ consists of 1 and all $x$ such that $5 \cdot 2^{n} \leqq x \leqq 5 \cdot 2^{n}+2^{n}-1$ for $n \geqq 1$. Also $\delta_{1}(A)=1 / 2$, $\delta_{1}(B)=1 / 3 . \quad \delta_{1}(A B)=1 / 6, \quad \delta_{1}(B A)=1 / 5, \quad d(A)=1 / 3, \quad d(B)=1 / 5$, $d(A B)=1 / 11$, and $d(B A)=1 / 9$.

\section{REFERENCES}

1. E. Artin and P. Scherk, On the sum of two sets of integers, Ann. of Math. vol. 44 (1943) pp. 138-142.

2. F. Behrend, Über numeri abundantes I, II, Preuss. Akad. Wiss. Sitzungsber. no. 21/23 (1932) pp. 322-328, no. 6 (1933) pp. 280-293.

3. - On sequences of numbers not divisible one by another, J. London Math. Soc. vol. 10 (1935) pp. 42-44.

4. - On sets of integers which contain no three terms in arithmetic progression, Proc. Nat. Akad. Sci. U.S.A. vol. 32 (1946) pp. 331-332.

5. A. S. Besicovitch, On the density of certain sequences of integers, Math. Ann. vol. 110 (1934) pp. 336-341.

6. - On the density of the sum of two sequences of integers, J. London Math. Soc. vol. 10 (1935) pp. 246-248.

7. R. C. Buck, The measure theoretic approach to density, Amer. J. Math. vol. 68 (1946) pp. 560-580.

8. E. F. Buck and R. C. Buck, $A$ note on finitely additive measures, Amer. J. Math. vol. 69 (1947) pp. 413-420.

9. L. P.-H. Cheo, $A$ remark on the $\alpha+\beta$ theorem, to appear in Proceedings of the American Mathematical Society.

10. - On the density of sets of Gaussian integers, to appear in Amer. Math. Monthly.

11. S. Chowla, On abundant numbers, J. Indian Math. Soc. N.S. vol. 1 (1934) pp. 41-44.

12. S. Chowla and J. Todd, The density of reducible integers, Canadian Journal of Mathematics vol. 1 (1949) pp. 297-299.

13. J. G. van der Corput, On sets of integers I, II, III, Neder. Akad. Wetensch. vol. 50 (1947) pp. 252-261, 340-350, 429-435.

14. —- On de Polignac's conjecture, Simon Stevin vol. 27 (1950) pp. 99-105. 
15. H. Davenport, Über numeri abundantes, Preuss. Akad. Wiss. Sitzungsber no. 26/29 (1933) pp. 830-837.

16. H. Davenport and P. Erdös, On sequences of positive integers, Acta Arithmetica vol. 2 (1936) pp. 147-151.

17. $\mathrm{H}$. Davenport and $\mathrm{H}$. Heilbronn, Note on a result in the additive theory of numbers, Proc. London Math. Soc. (2) vol. 43 (1937) pp. 142-151.

18. F. J. Dyson, $A$ theorem on the densities of sets of integers, J. London Math. Soc. vol. 20 (1945) pp. 8-14.

19. P. Erdös, On the density of the abundant numbers, J. London Math. Soc. vol. 9 (1934) pp. 278-282.

20. - On the density of some sequences of integers I, II, J. London Math. Soc. vol. 10 (1935) pp. 120-125; vol. 12 (1937) pp. 7-11.

21. - Note on sequences of integers no one of which is divisible by any other, J. London Math. Soc. vol. 10 (1935) pp. 126-128.

22. - On the arithmetical density of the sum of two sequences one of which forms a basis for the integers, Acta Arithmetica vol. 1 (1936) pp. 197-200.

23. - On a problem of Chowla and some related problems, Proc. Cambridge Philos. Soc. vol. 32 (1936) pp. 530-540.

24. - A generalization of a theorem of Besicovitch, J. London Math. Soc. vol. 11 (1936) pp. 92-98.

25. - On the easier Waring problem for powers of primes I, Proc. Cambridge Philos. Soc. vol. 33 (1937) pp. 6-12.

26. - On the asymptotic density of the sum of two sequences one of which forms a basis for the integers II, Travaux de l'Institut Mathématique de Tblissi vol. 3 (1938) pp. 217-224.

27. - On the asymptotic density of the sum of two sequences, Ann. of Math. vol. 43 (1942) pp. 65-68.

28. - On the density of some sequences of integers, Bull. Amer. Math. Soc. vol. 54 (1948) pp. 685-692.

29. - Some asymptotic formulas in number theory, J. Indian Math. Soc. N.S. vol. 12 (1948) pp. 75-78.

30. P. Erdös and I. Niven, The $\alpha+\beta$ hypothesis and related problems, Amer. Math. Monthly vol. 53 (1946) pp. 314-317.

31. P. Erdös and P. Turán, On some sequences of integers, J. London Math. Soc. vol. 11 (1936) pp. 261-264.

32. W. Feller and E. Tornier, Mengentheoretische Untersuchungen von Eigenschaften der Zahlreihe, Math. Ann. vol. 107 (1932) pp. 188-232.

33. E. Fogels, Zur Arithmetik quadratischer Zahlenkörper, Universität in Riga. Wissenschaftliche Abhandlungen. Klasse der Mathematischen Abteilung vol. 1 (1943) pp. 23-47.

34. H. Heilbronn, On an inequality in the elementary theory of numbers, Proc. Cambridge Philos. Soc. vol. 33 (1937) pp. 207-209.

35. M. Kac, Probability methods in some problems of analysis and number theory, Bull. Amer. Math. Soc. vol. 55 (1949) pp. 641-665.

36. A. Khintchine, Zur additiven Zahlentheorie, Rec. Math. (Mat. Sbornik) vol. 39 (1932) pp. 27-34.

37. E. Landau, Verschärfung eines Romanoffschen Satzes, Acta Arithmetica vol. 1 (1935) pp. 43-61.

38. — Über eine neuere Fortschritte der additiven Zahlentheorie, Cambridge Tract no. 35, 1937. 
39. B. Lepson, Certain best possible results in the theory of Schnirelmann density, Proceedings of the American Mathematical Society vol. 1 (1950) pp. 592-594.

40. U. V. Linnik, On Erdös's theorem on the addition of numerical sequences, Rec. Math. (Mat. Sbornik) N.S. vol. 10 (1942) pp. 67-78.

41. H. B. Mann, $A$ proof of the fundamental theorem on the density of sums of sets of positive integers, Ann. of Math. vol. 43 (1942) pp. 523-527.

42. - On the number of integers in the sum of two sets of positive integers, Pacific Journal of Mathematics vol. 1 (1951) pp. 249-253.

43. L. Mirsky, A property of square-free integers, J. Indian Math. Soc. N.S. vol. 13 (1949) pp. 1-3.

44. L. Moser, On sets of integers which contain no three in arithmetical progression, Part I of Ph.D. dissertation, University of North Carolina, 1950.

45. I. Niven, $A$ binary operation on sets of positive integers, Bull. Amer. Math. Soc. Abstract 56-5-415.

46. - Sets of integers of density zero, Proceedings of the International Congress, Cambridge, 1950.

47. H.-H. Ostmann, Beweis einer Vermutung itber die asymptotische Dichte und Verscharfung einer Abschatzung filr die Dichte der Summe zweier Zählenmengen, Deutsche Mathematik vol. 6 (1941) pp. 213-247.

48. — Über die Dichten additiv komponierter Zahlenmengen, Archiv der Mathematik vol. 1 (1949) pp. 393-401.

49. - Verfeinerte Lösung der asymptotischen Dichtenaufgabe, J. Reine Angew. Math. vol. 187 (1950) pp. 183-188.

50. - Über die Anzahl der Elemente von Summenmengen, J. Reine Angew. Math. vol. 187 (1950) pp. 222-230.

51. G. P6lya, Untersuchungen ulber Litcken and Singularitäten von Potenzreihen, Math. Zeit. vol. 29 (1929) pp. 549-640.

52. H. Rohrbach, Beweis einer zahlentheoretische Ungleichung, J. Reine Angew. Math. vol. 177 (1937) pp. 193-196.

53. - Einige neuere Untersuchungen über die Dichte in der additiven Zahlentheorie, Jber. Deutschen Math. Verein. vol. 48 (1938) pp. 199-236.

54. N. P. Romanoff, Über einige Sätze der additiven Zahlentheorie, Math. Ann. vol. 109 (1934) pp. 668-678.

55. R. Salem and D. C. Spencer, The influence of gaps on density of integers, Duke Math. J. vol. 9 (1942) pp. 855-872.

56. - On sets of integers which contain no three terms in arithmetic progression, Proc. Nat. Acad. Sci. U.S.A. vol. 28 (1942) pp. 561-563.

57. - On sets which do not contain a given number of terms in arithmetic progression, Nieuw Archief voor Wiskunde vol. 23 (1950) pp. 133-143.

58. L. Schnirelmann, Über additive Eigenschaften von Zahlen, Math. Ann. vol. 107 (1932) pp. 649-690.

59. I. J. Schoenberg, On asymptotic distribution of arithmetical functions, Trans. Amer. Math. Soc. vol. 39 (1936) pp. 315-330.

60. S. Selberg, Note on the distribution of the integers $a x^{2}+b y^{2}+c^{z^{2}}$, Archiv for Mathematik og Naturvidenskab vol. 50 (1949) pp. 65-69.

61. H. N. Shapiro, Some remarks on a theorem of Erdös concerning asymptotic density, Proceedings of the American Mathematical Society vol. 1 (1950) pp. 590-592.

UNIVERSITY OF OREGON 